\title{
Nodular Regenerative Hyperplasia of the Liver: A Rare Vascular Complication in Systemic Sclerosis
}

\author{
Laura Graf, Rucsandra Dobrota, Suzana Jordan, Lukas Martin Wildi, Oliver Distler, \\ and Britta Maurer
}

ABSTRACT. Objective. To investigate nodular regenerative hyperplasia (NRH) as a vascular complication of systemic sclerosis (SSc) with microvasculopathy as a common denominator.

Methods. Cases of SSc-NRH were identified by systematic literature review and by screening the Zurich cohort. NRH had to be diagnosed by liver biopsy.

Results. Literature review retrieved 22 cases. In our cohort, $1.4 \%$ of patients with SSc were diagnosed with NRH. Most had vasculopathy, were positive for anticentromere antibodies, had elevated alkaline phosphatase and gamma-glutamyl transferase levels, normal liver morphology on ultrasound yet increased stiffness on ultrasound elastography, and had portal hypertension.

Conclusion. NRH might represent a rare yet potentially life-threatening vascular complication in SSc.

(First Release November 1 2017; J Rheumatol 2018;45:103-6; doi:10.3899/jrheum.170292)

Key Indexing Terms:

NODULAR REGENERATIVE HYPERPLASIA

Nodular regenerative hyperplasia (NRH) of the liver is a rare and poorly understood liver disease. NRH is histologically defined by diffuse micronodular transformation without fibrous septa. Lack of perinuclear collagen tissue distinguishes NRH from typical regenerative nodules in the cirrhotic liver. So far, there are only about 460 reported cases, and most of the knowledge of NRH is based upon case reports rather than systematic population studies. Patients with NRH may remain asymptomatic; however, in at least $50 \%$ of reported cases, potentially life-threatening complications occur. Although the etiology is unknown, it is hypothesized that NRH develops as a result of microvascular alterations $1,2,3,4$. Data indicate that damage of endothelial cells might play an important role. Clinical complications of NRH comprise manifestations of portal hypertension such as splenomegaly, ascites, and esophageal or gastric varices. Transaminases might be normal or slightly elevated, whereas cholestatic measures are often more significantly increased $^{2,3,4}$.

Among the autoimmune disorders, systemic sclerosis (SSc) in particular has been suggested to be associated with

From the Department of Rheumatology, University Hospital Zurich, Zurich, Switzerland.

L. Graf, cand.med., Department of Rheumatology, University Hospital Zurich; R. Dobrota, MD, Department of Rheumatology, University

Hospital Zurich; S. Jordan, MD, Department of Rheumatology, University Hospital Zurich; L.M. Wildi, MD, Department of Rheumatology,

University Hospital Zurich; O. Distler, MD, Department of Rheumatology,

University Hospital Zurich; B. Maurer, MD, Department of Rheumatology,

University Hospital Zurich.

Address correspondence to Dr. B. Maurer, Department of Rheumatology,

University Hospital Zurich, Gloriastrasse 25, 8091 Zurich, Switzerland.

E-mail:britta.maurer@usz.ch

Accepted for publication September 8, 2017.

\section{VASCULOPATHY SYSTEMIC SCLEROSIS}

$\mathrm{NRH}^{3,5}$. In SSc, microvascular injury, including damage of endothelial cells, is considered one of the earliest pathologic events, followed by inflammation and fibrosis ${ }^{6}$. Similar mechanisms are suspected of being operative in $\mathrm{NRH}^{1,3}$. Therefore, it might be hypothesized that NRH represents a yet unidentified vascular alteration in SSc and possibly other autoimmune diseases with associated microvasculopathy, such as systemic lupus erythematosus or rheumatoid arthritis ${ }^{1,3,7,8,9}$. So far, the prevalence of SSc-NRH is unknown, and the clinical phenotype of patients with SSc-NRH has not yet been characterized systematically. In addition, the prognosis of SSc-NRH remains elusive. Therefore, the aim of our study was to investigate the prevalence and the clinical phenotype of NRH in our SSc cohort.

\section{MATERIALS AND METHODS}

First, we performed an electronic search by systematically screening the databases Pubmed, Medline, Google Scholar, and the Cochrane Library for available literature. Combinations of medical subject headings and free text words related to "systemic sclerosis, scleroderma, nodular regenerative hyperplasia" were used. Articles published in English, Spanish, Italian, French, and German were considered from inception of the databases up to December 2016. The search results were supplemented by articles found through manually screening the reference lists of identified studies. Studies were included if they were original case reports or series and reported on biopsy-proven SSc-NRH. After removal of duplicates, the search results were screened for eligibility by a team of 2 reviewers (LG/BM) sharing the retrieved citations. In case of disagreement, a third party (DO) served as referee. Next, we screened our Zurich SSc cohort, which comprised 278 patients with established SSc fulfilling the American College of Rheumatology (ACR)/European League Against Rheumatism (EULAR) criteria $^{10}$ at the time of the data analysis. In accordance with international guidelines, the diagnosis of NRH had to be established by liver biopsy showing a characteristic diffuse micronodular transformation without fibrous septa $^{4}$. SSc characteristics were derived from the local SSc database in Zurich in accordance with EULAR Scleroderma Trials and Research group

Personal non-commercial use only. The Journal of Rheumatology Copyright $\odot$ 2018. All rights reserved. 
(EUSTAR) recommendations ${ }^{11}$. Information on $\mathrm{NRH}$ was extracted from the patients' charts. Patients with any connective tissue disease other than SSc or not fulfilling the ACR/EULAR 2013 criteria were excluded. All patients signed informed consent according to the Declaration of Helsinki, and the Cantonal Ethics Committee Zurich approved the study (PB2016_01515). To identify additional nonpublished studies of patients with SSc-NRH through expert opinion, we repeatedly conducted a questionnaire-based inquiry by contacting $>180$ EUSTAR centers worldwide by e-mail between February 2015 and January 2016. However, because the eligibility of cases for our study was based on biopsy-proven NRH, no additional cases could be retrieved. For the statistical analysis, IBM SPSS software version 20 was used. Normal distribution of data was examined using the Kolmogorov-Smirnov test. For parametric nonrelated data, expressed as mean \pm standard error of the mean (SEM), the unpaired 2-tailed t test was used. Nonparametric nonrelated data, expressed as median (Q1, Q3), were analyzed using the Mann-Whitney $\mathrm{U}$ test. $\mathrm{P}$ values $<0.05$ were considered statistically significant.

\section{RESULTS}

The literature review provided 1698 citations. After screening and checking for duplicates, 17 reports $1,3,7,8,9,12-25$ evaluating 22 patients with SSc-NRH remained. In the Zurich cohort, 4 out of 278 patients with established SSc were diagnosed with biopsy-proven NRH (Figure 1), resulting in a prevalence of symptomatic NRH of $1.4 \%$. An additional question- naire-based EUSTAR inquiry did not retrieve additional results based on eligibility. The majority of those 26 patients were women $(75 \%)$ with an average age of $46 \pm 11.9$ at diagnosis of SSc. Mean disease duration of SSc was $6.5 \pm 5.6$ years when NRH was diagnosed. NRH occurred both in diffuse $(\mathrm{n}=12,54.5 \%)$ and limited cutaneous SSc ( $=8,36.4 \%$ ), as well as in 2 patients without skin involvement $(9.1 \%)$. In 4 published cases, the extent of skin involvement was not reported. Of note, in most patients, vascular features of SSc were present at the time of NRH diagnosis, including digital ulcers (ever $100 \%$, active $71.4 \%$ ), an active pattern on nailfold capillaroscopy (100\%), and pulmonary hypertension $(63 \%)$. The most prevalent autoantibodies were anticentromere $(50 \%)$ and anti-U1nRNP $(33 \%)$. In most patients, an elevation of alkaline phosphatase (AP, 85\%) and gammaglutamyl transferase (GGT, 60\%) was observed, whereas transaminases were not increased. Melena and hematemesis occurred in $71 \%$ and $56 \%$ of patients, respectively. Ultrasound detected ascites $(75 \%)$ and splenomegaly $(82 \%)$, but no pathologic liver morphology. However, an increased stiffness $\left[\mathrm{kPa} 14_{(5,21)}\right.$, reference $\left.\leq 7.5 \mathrm{kPa}\right]$ was diagnosed by ultrasound elastography (FibroScan by EchoSens; 75\%).

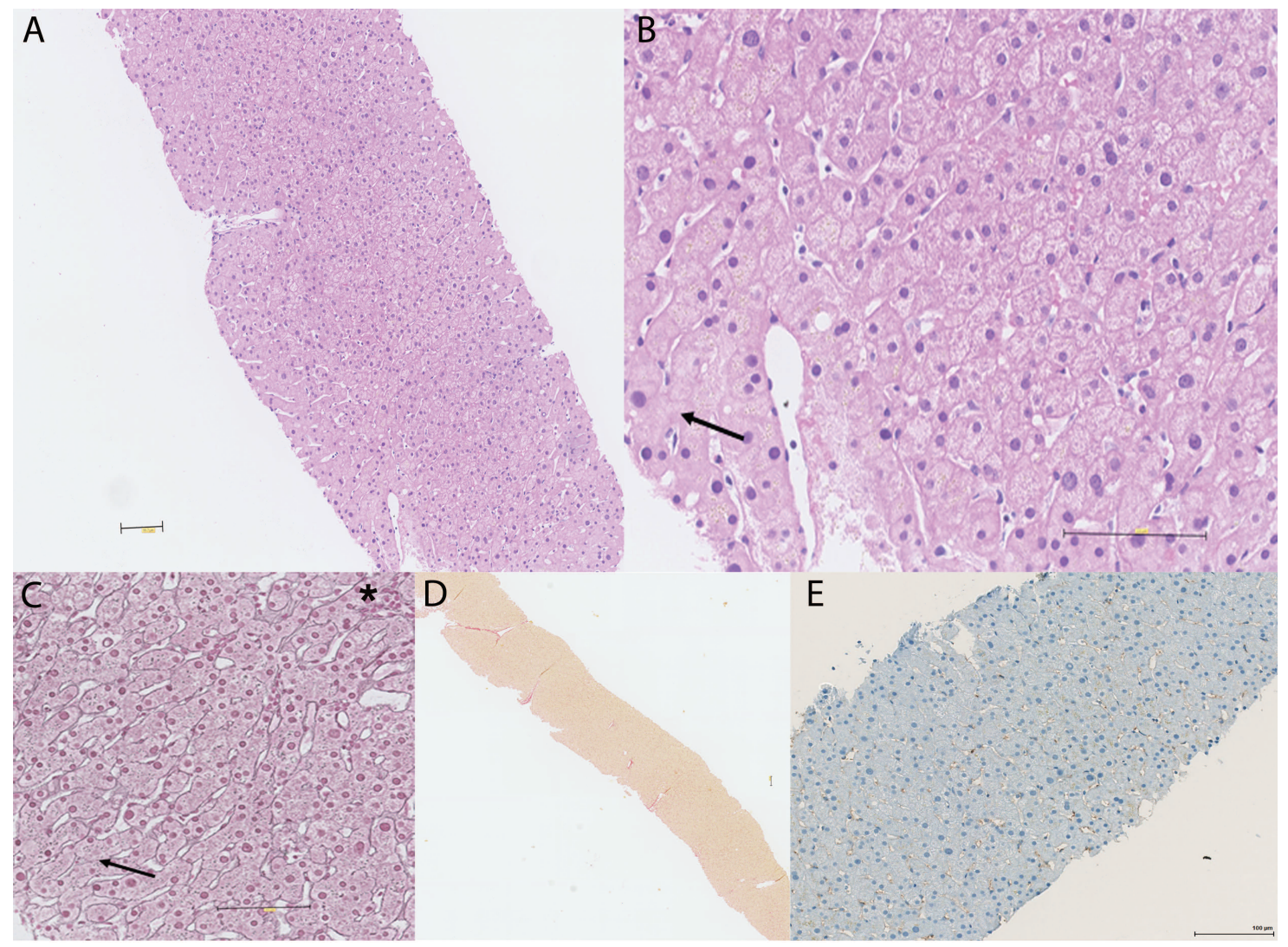

Figure 1. Nodular regenerative hyperplasia on liver biopsy (A) shows subtle nodularity from liver parenchyma stained with H\&E; panels B and C demonstrate discrete hypotrophic hepatocyte plates (asterisk) juxtaposed to slightly hypertrophic hepatocyte plates (arrow) using H\&E and reticulin staining; in (D), Sirius Red staining demonstrates the absence of significant fibrosis; (E) highlights the relative rarefaction of capillaries visualized by immunohistochemical staining of endothelial cells (von Willebrand factor-positive, brown DAB staining).

Personal non-commercial use only. The Journal of Rheumatology Copyright $@$ 2018. All rights reserved 
Portal hypertension, defined as hepatic venous pressure gradient $\geq 5 \mathrm{mmHg}$ measured by catheter during transjugular liver biopsy under fluoroscopic guidance, was diagnosed in $90 \%$ of patients and had esophageal varices $(77 \%)$ and variceal hemorrhage $(58 \%)$ as main complications, which were the reasons for which most patients underwent liver biopsy. The presence of hepatitis B, C, or human immunodeficiency virus, and primary biliary cholangitis were excluded by serologic tests and liver biopsies. The main characteristics of all 26 patients (as far as available) are provided in Table 1.

\section{DISCUSSION}

The data derived from our own SSc cohort as well as from the literature support the hypothesis of $\mathrm{NRH}$ as a rare vascular complication of SSc, especially in anticentromerepositive patients. Consistently, all patients had a history of digital ulcers, with $70 \%$ even having active ulcers at the time of NRH diagnosis. Pulmonary arterial hypertension (PAH), renal crisis, and active nailfold capillaroscopy, all vascular complications of SSc, were also present in most patients from our local cohort. Immunosuppression and treatment with cytotoxic agents, particularly within the context of autoimmune diseases, and organ or stem cell transplantation, is discussed as another contributing factor for the development of $\mathrm{NRH}^{2,12}$. Although $28 \%$ of patients $(5 / 18$, no data available for 7) were treated with immunosuppressive agents (including corticosteroids $\leq 10 \mathrm{mg} / \mathrm{d}$, mycophenolate mofetil, rituximab, cyclosporine, methotrexate, cyclophosphamide, and human immunoglobulins), none were under treatment with azathioprine, which has been predominantly suggested for patients who either have autoimmune diseases or have undergone organ transplantation, and particularly in cases with decreased thiopurine methyltransferase activity $2,26,27$. As our limited data suggest, the prevalence in SSc might be higher because in most patients with $\mathrm{NRH}$, only late-stage complications owing to portal hypertension lead to the diagnosis. This is especially true because liver enzymes (apart from measures of cholestasis) and ultrasound findings are not pathologic in most patients. Ultrasound elastography or hepatic magnetic resonance imaging findings might raise suspicion, however. Even then, liver biopsies are often not performed before the onset of bleeding complications and/or the development of ascites. Therefore, we suggest that an extended diagnostic investigation be performed in patients with SSc who have persisting elevation of GGT and AP in the presence of other risk factors such as female sex, established SSc, microvasculopathy (peripheral, PAH), and positivity for anticentromere or anti-U1nRNP antibodies. This assessment should include the performance of a liver ultrasound to screen for signs of portal hypertension, ultrasound elastography to evaluate the presence of fibrosis/cirrhosis, and in cases of upper gastrointestinal bleeding, a gastroscopy. Depending on the obtained results, a liver
Table 1. Patients' characteristics, $\mathrm{n}=26$.

\begin{tabular}{|c|c|c|}
\hline Characteristics & $\mathrm{n}(\%)$ & Median (IQR) \\
\hline \multicolumn{3}{|l|}{ Peripheral vasculopathy } \\
\hline Raynaud phenomenon & $19 / 19(100)$ & N/A \\
\hline Digital ulcers ever & 7/7 (100) & N/A \\
\hline Active digital ulcers & $5 / 7(71)$ & N/A \\
\hline \multicolumn{3}{|l|}{ Nailfold capillaroscopy } \\
\hline Early & $0 / 5(0)$ & N/A \\
\hline Active & $5 / 5(100)$ & N/A \\
\hline Late & $0 / 5(0)$ & N/A \\
\hline \multicolumn{3}{|l|}{ Laboratory variables } \\
\hline ANA & $12 / 13(92)$ & N/A \\
\hline Anticentromere antibodies & $6 / 12(50)$ & N/A \\
\hline Anti-Scl-70 & $1 / 8(12.50)$ & N/A \\
\hline Anti-RNA-polymerase III & $1 / 6(17)$ & N/A \\
\hline Anti-U1nRNP & $2 / 6(33)$ & N/A \\
\hline AST elevated, U/1 & $3 / 11(27)$ & $38(27,89)$ \\
\hline \multicolumn{3}{|l|}{ Organ involvement } \\
\hline \multicolumn{3}{|l|}{ Lung } \\
\hline Dyspnea present & $9 / 9(100)$ & N/A \\
\hline Pulmonary hypertension $^{\mathrm{a}}$ & $5 / 8(63)$ & N/A \\
\hline Lung fibrosis ${ }^{\mathrm{b}}$ & $6 / 9(67)$ & N/A \\
\hline \multicolumn{3}{|l|}{ Gastrointestinal tract } \\
\hline Esophageal symptoms & $10 / 10(100)$ & N/A \\
\hline Intestinal symptoms & $6 / 8(75)$ & N/A \\
\hline Stomach symptoms & $4 / 7(57)$ & N/A \\
\hline Melena & $5 / 7(71)$ & N/A \\
\hline Hematemesis & $5 / 9(56)$ & N/A \\
\hline \multicolumn{3}{|l|}{ Kidney } \\
\hline Renal crisis & $2 / 6(33)$ & N/A \\
\hline Liver & & N/A \\
\hline \multicolumn{3}{|l|}{ Ultrasound } \\
\hline Liver size normal & $6 / 8(75)$ & N/A \\
\hline Surface smooth & $3 / 4(75)$ & N/A \\
\hline Parenchyma homogeneous & $3 / 4(75)$ & N/A \\
\hline Focal lesions & $0 / 5(0)$ & N/A \\
\hline Portal vein flow normal & $4 / 5(80)$ & N/A \\
\hline Ascites & $6 / 8(75)$ & N/A \\
\hline Splenomegaly & $9 / 11(82)$ & N/A \\
\hline \multicolumn{3}{|l|}{ Ultrasound elastography } \\
\hline Increased stiffness, $\mathrm{kPa}$ & $3 / 4(75)$ & $14(5,21)$ \\
\hline \multicolumn{3}{|l|}{ Complications } \\
\hline Portal hypertension ${ }^{\mathrm{c}}, \mathrm{mmHg}$ & $9 / 10(90)$ & $20.5(6,26)$ \\
\hline Gastric varices, by gastroscopy & $2 / 6(33)$ & N/A \\
\hline Esophageal varices, by gastroscopy & $10 / 13(77)$ & N/A \\
\hline Variceal hemorrhage, by gastroscopy & $7 / 12(58)$ & N/A \\
\hline
\end{tabular}

aDefined as mPAP $>45 \mathrm{mmHg}$ on echocardiography or $>25 \mathrm{mmHg}$ on right heart catheterization. ${ }^{\mathrm{b}}$ Defined as $\mathrm{FVC}<60$ or $\mathrm{FVC}<70$ and the presence of lung fibrosis on HRCT. ${ }^{c}$ Defined as hepatic venous pressure gradient $\geq 5$ $\mathrm{mmHg}$. For nominal variables, the absolute and relative frequency is shown. All other variables are presented as median with first and third quartiles. The percentages indicate positive findings in the absolute number of patients for whom the respective information was available. Italics indicate most relevant findings. Definition of items and organ manifestation are according to the EUSTAR registry ${ }^{11}$, unless otherwise specified. ANA: antinuclear antibodies; EUSTAR: EULAR Scleroderma Trials and Research group; HRCT: high-resolution computed tomography; N/A: not available; AST: aspartate aminotransferase; mPAP: mean pulmonary arterial pressure; FVC: forced vital capacity; EULAR: European League Against Rheumatism.

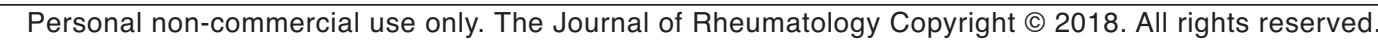


biopsy should be performed to establish the final diagnosis by simultaneously excluding the presence of primary biliary cholangitis (PBC), which occasionally occurs in patients with (anticentromere-positive) SSc, although most often it is additionally characterized by the presence of antimitochondrial and anti-M2 antibodies ${ }^{28}$.

NRH might represent a rare yet clinically important, potentially life-threatening complication in patients with SSc, especially in those with prominent vascular features and positivity for anticentromere antibodies. Mildly to moderately elevated levels of AP and GGT (i.e., 2-3x upper limit of normal), ascites, as well as splenomegaly by ultrasound $(>11 \times 4 \times 7 \mathrm{~cm})$ and increased stiffness by ultrasound elastography $(>7.5 \mathrm{kPa})$ might indicate the presence of $\mathrm{NRH}$ as another important differential diagnosis to $\mathrm{PBC}$, as illustrated by our case series. Therefore, if suspected, the diagnosis should be confirmed by liver biopsy.

\section{ACKNOWLEDGMENT}

The authors thank Dr. Ewerton Marques Maggio, Department of Pathology, University Hospital Zurich, for performing the histologic and immunohistochemical analyses of the liver biopsies carried out at the University Hospital Zurich. The authors also thank the members of the EUSTAR network for support with the questionnaire-based inquiry on SSc-NRH.

\section{REFERENCES}

1. Wanless IR. Micronodular transformation (nodular regenerative hyperplasia) of the liver: a report of 64 cases among 2,500 autopsies and a new classification of benign hepatocellular nodules. Hepatology 1990;11:787-97.

2. Steiner P. Nodular regenerative hyperplasia of the liver. Am J Pathol 1959;35:943-53.

3. Hartleb M, Gutkowski K, Milkiewicz P. Nodular regenerative hyperplasia: evolving concepts on underdiagnosed cause of portal hypertension. World J Gastroenterol 2011;17:1400-9.

4. Arvanitaki M, Adler M. Nodular regenerative hyperplasia of the liver. A review of 14 cases. Hepatogastroenterology 2001; 48:1425-9.

5. Ziol M, Poirel H, Kountchou GN, Boyer O, Mohand D, Mouthon L, et al. Intrasinusoidal cytotoxic CD8+ T cells in nodular regenerative hyperplasia of the liver. Hum Pathol 2004;35:1241-51.

6. Allanore Y, Simms R, Distler O, Trojanowska M, Pope J, Denton CP, et al. Systemic sclerosis. Nat Rev Dis Primers 2015:15002.

7. Matsumoto T, Kobayashi S, Shimizu H, Nakajima M, Watanabe S, Kitami N, et al. The liver in collagen diseases: pathologic study of 160 cases with particular reference to hepatic arteritis, primary biliary cirrhosis, autoimmune hepatitis and nodular regenerative hyperplasia of the liver. Liver 2000;20:366-73.

8. Vaiphei K, Bhatia A, Sinha SK. Liver pathology in collagen vascular disorders highlighting the vascular changes within portal tracts. Indian J Pathol Microbiol 2011;54:25-31.

9. Perez Ruiz F, Orte Martinez FJ, Zea Mendoza AC, Ruiz del Arbol L, Moreno Caparros A. Nodular regenerative hyperplasia of the liver in rheumatic diseases: report of seven cases and review of the literature. Semin Arthritis Rheum 1991;21:47-54.

10. van den Hoogen F, Khanna D, Fransen J, Johnson SR, Baron M, Tyndall A, et al. 2013 classification criteria for systemic sclerosis: an American College of Rheumatology/European League Against Rheumatism collaborative initiative. Ann Rheum Dis 2013; 72:1747-55.

11. Meier FM, Frommer KW, Dinser R, Walker UA, Czirjak L, Denton
$\mathrm{CP}$, et al. Update on the profile of the EUSTAR cohort: an analysis of the EULAR Scleroderma Trials and Research group database. Ann Rheum Dis 2012;71:1355-60.

12. Cadranel JF, Grippon P, Wechsler B, Bidegaray E, Karkouche B, Opolon P. [The CRST syndrome and nodular regenerative hyperplasia of the liver. A case]. [Article in French] Presse Med 1987;16:1656.

13. Friguet JL, Deugnier Y, Messner M, Lauvin R, Launois B, Brissot P, et al. [Regenerative nodular hyperplasia in scleroderma: a new case]. [Article in French] Gastroenterol Clin Biol 1984;8:979.

14. Agard C, Ponge T, Mahé B, Barrier J. [Lymphocytic lymphoma and regenerative liver nodular hyperplasia in systemic scleroderma]. [Article in French] Rev Med Interne 2000;21:301-3.

15. Kaburaki J, Kuramochi S, Fujii T, Kuwana M, Tojo T, Ikeda Y, et al. Nodular regenerative hyperplasia of the liver in a patient with systemic sclerosis. Clin Rheumatol 1996;15:613-6.

16. Riviere E, Vergniol J, Reffet A, Lippa N, Le Bail B, de Ledinghen V. Gastric variceal bleeding uncovering a rare association of CREST syndrome, primary biliary cirrhosis, nodular regenerative hyperplasia and pulmonary hypertension. Eur J Gastroenterol Hepatol 2010;22:1145-8.

17. Russell ML, Kahn HJ. Nodular regenerative hyperplasia of the liver associated with progressive systemic sclerosis: a case report with ultrastructural observation. J Rheumatol 1983;10:748-52.

18. García Díaz JD, Praga Terente M, Colina Ruizdelgado F, Lizasoaín Hernández M, Mesa Latorre J. [Nodular regenerative hyperplasia of the liver associated with a CREST syndrome with multiorgan involvement]. [Article in Spanish] An Med Interna 1989;6:203-6.

19. McMahon RF, Babbs C, Warnes TW. Nodular regenerative hyperplasia of the liver, CREST syndrome and primary biliary cirrhosis: an overlap syndrome? Gut 1989;30:1430-3.

20. Lurie B, Novis B, Bank S, Silber W, Botha JB, Marks IN. CRST syndrome and nodular transformation of the liver. A case report. Gastroenterology 1973;64:457-61.

21. Colina F, Alberti N, Solis JA, Martinez-Tello FJ. Diffuse nodular regenerative hyperplasia of the liver (DNRH). A clinicopathologic study of 24 cases. Liver 1989;9:253-65.

22. Tsuneyama K, Harada K, Katayanagi K, Watanabe K, Kurumaya H, Minato $\mathrm{H}$, et al. Overlap of idiopathic portal hypertension and scleroderma: Report of two autopsy cases and a review of literature. J Gastroenterol Hepatol 2002;17:217-23.

23. Mendel A. Nodular regenerative hyperplasia of the liver presenting with ascites in a woman with limited systemic sclerosis. Univ West Ont Med J 2011;80 Suppl S1:11-3.

24. Kamel G, Espiritu J, Di Bisceglie AM, Chen G, Syed R, Nayak R. Pulmonary hypertension in a patient with non-cirrhotic portal hypertension and scleroderma sine scleroderma: a case report. J Med Case Rep 2016;4:170-2.

25. Morris JM, Oien KA, McMahon M, Forrest EH, Morris J, Stanley $\mathrm{AJ}$, et al. Nodular regenerative hyperplasia of the liver: survival and associated features in a UK case series. Eur J Gastroenterol Hepatol 2010;22:1001-5.

26. Blogowski W, Marlicz W, Smereczynski A, Lawniczak M, Lewosiuk A, Starzynska T. Nodular regenerative liver hyperplasia as a complication of azathioprine-containing immunosuppressive treatment for Crohn's disease. Immunopharmacol Immunotoxicol 2011;33:398-402.

27. de Boer NK, Mulder CJ, van Bodegraven AA. Nodular regenerative hyperplasia and thiopurines: the case for level-dependent toxicity. Liver Transpl 2005;11:1300-1.

28. Zheng B, Vincent C, Fritzler MJ, Senecal JL, Koenig M, Joyal F. Prevalence of systemic sclerosis in primary biliary cholangitis using the new ACR/EULAR classification criteria. J Rheumatol 2017;44:33-9. 